\title{
Identification of Determinant Factors of Health-Related Quality of Life in Iranian Urban Population
}

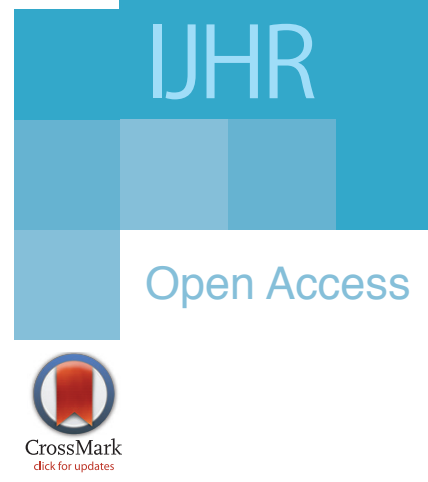

\author{
Sajad Vahedi ${ }^{1}$, Aziz Rezapour ${ }^{2}$, Masoud Arefnezhad ${ }^{3 *}$, Sajad Lak ${ }^{4}$, Fatemeh \\ Bastami ${ }^{5}$ \\ ${ }^{1}$ Health Management and Economics Research Center, Iran University of Medical sciences, Tehran, Iran. ${ }^{2}$ School \\ of Health Management and Information Sciences, Iran University of Medical sciences, Tehran, Iran. ${ }^{3}$ Department of \\ Healthcare Management, Zabol University of Medical Sciences, Zabol, Iran. ${ }^{4}$ Department of Health Care Management, \\ School of Medicine, Science and Research Branch, Islamic Azad University, Tehran, Iran. ${ }^{5}$ Department of Health \\ Education and Promotion, Faculty of Health, Isfahan University of Medical Sciences, Isfahan, Iran.
}

First Published online March 30, 2016

\begin{abstract}
Background and Objectives: Identification of health-related quality of life (HRQoL) could provide important implications for health policy makers. While quality of life is well addressed in the Iranian context, studies on HRQoL are relatively scarce. This study was therefore designed to explore HRQoL and identify its determinant factors among population of an Iranian metropolis.

Methods: This cross-sectional study was conducted in Shiraz, the capital of the Iranian Fars province. Applying a multistage sampling method, a random sample 1610 of individuals of age $>18$ years were surveyed, using an adapted and validated version of HRQoL Short-Form 36 questionnaire. Data was summarized by descriptive statistical methods. Mean valued were compared using $t$ test and Search Results

Image result for Analysis of variance (ANOVA) and logistic regression was used to identify the determinants of HRQoL.

Findings: Age, gender, employment, education level, physical activity, and smoking were identified as significant determinants of physical HRQoL.

Conclusions: Interventions to enhance HRQoL should be focused on females and elderly, primarily. Public educations focused on life style improvement would be an affordable and effective strategy to enhance HRQoL. Our results also encourage further of large-scale studies to enable incorporation of HRQoL concept into national policy-making.
\end{abstract}

Keywords: Health-related quality of life, Survey, Life style, Public health

\section{Background and Objectives}

More than 30 years ago, Alma-Ata Declaration signatories noted that "health for all" would contribute to a better quality of life (QOL) and global peace and security. ${ }^{1}$ The ultimate goal of health care is to improve, restore, and maintain the individuals' health, so that they can enjoy a high-quality life. An important health policy concern in many countries is the extent to which the improvement of QOL is keeping pace with an increased life expectancy. ${ }^{2}$ QOL has been defined by the World Health Organization (WHO) as "an individual's perception of their posi-

${ }^{*}$ Corresponding Author: Masoud Arefnezhad, Department of Healthcare Management, Zabol University of Medical Sciences, Zabol, Iran, Tel: +98 9153425651, Email: masoudarefnezhad@gmail.com tion in life in the context of the culture and value systems in which they live in relation to their goals, expectations, standards, and concerns." 3

Health is one of the major components of well-being, directly impacting other aspects of life. Given that, the concept of health-related quality of life (HRQoL) has been developed to bring a particular focus on the influence of health on the entire life and its quality. HRQoL, is defined as the perception of an individual or a population group towards their physical and mental health across the time. ${ }^{4,5}$ Being an intrinsically multidimensional construct, HRQoL can reflect many hidden variables influencing health-related outcomes. Therefore, adopting HRQoL as a major index of health would be more informative in police-making than the naïve concepts such as mortality 
and morbidity rate. ${ }^{4} \mathrm{HRQ}$ oL has emerged as an important domain of study and attracted an ever-increasing interest over the past two decades..$^{5,6}$ In Iran, however, the concept is relatively new and few studies have investigated the determinants of HRQoL. ${ }^{5}$ Hence, the present study aimed to explore the socioeconomic determinants of $\mathrm{HRQOL}$ among a sample of Iranian Urban population taken from a metropolitan city.

\section{Methods}

\section{Study Design and Sample}

This cross-sectional study was conducted among population of Shiraz, the capital of Fars province of Iran. All the individuals above 18 years old were considered as the study population. A 4-stage sampling strategy was adopted. First, the municipality areas were regarded as classes and the sample size was determined in proportion to the population of each class. Each municipality areas were then divided into 10 residential blocks. The households were then selected by systematic sampling, and finally 1610 individuals were picked out through KISH grid method.

\section{Study Tool}

A translated version of HRQoL Short-Form 36 (SF-36) which was adapted to the Iranian context ${ }^{7}$ was used as the study instrument. The scale account for 8 subscales evaluating the health status of an individual from both physical and mental aspects. The first four subscales; i.e., Physical Functioning (PF), Role-Physical (RP), Bodily Pain (BP), and General Health $(\mathrm{GH})$, are related to physical health and yield the Physical Component Summary (PCS) score. The next four subscales; i.e., Vitality (VT), Social Functioning (SF), Role-Emotional (RE), and Mental Health (MH), are related to mental health and yield the Mental Component Summary (MCS) score. The study participants were asked to respond to the questionnaire items using a numerical scale. These responses were then coded and assigned a score on a scale of 0-100. Higher scores represented a more favorable perception of physical and mental health.

\section{Statistical Analysis}

The difference in mean values was tested by $t$ test and analysis of variance (ANOVA). Determinants of HRQoL were predicted using multiple logistic regression by dividing the subjects into 2 groups: those with PCS and MCS scores were equal to or higher than the mean and those whose with the same scores below the mean. Socio-demographic factors, such as gender, age, marital status, income, education level, physical activity, and smoking habit were considered as independent variables. The data were analyzed using the SPSS version 21 software package.

Ethical Issues

The study was approved by the Ethics Committee of the Shiraz University of Medical Sciences. The participants were briefed about the goals of the study and their informed consent was obtained.

\section{Results}

Out of 1610 distributed questionnaires 1568 valid completed questionnaires were returned yielding a response rate of $97.3 \%$. Table 1 presents the characteristics of the study subjects. The age of the subjects ranges from 18 to 88 years averaging at $36.88(S D=15.41)$ years. While

Table 1. Socio-Demographic Characteristics of the Participants $(n=1568)$

\begin{tabular}{|c|c|c|}
\hline Variables & No. & $\%$ \\
\hline \multicolumn{3}{|l|}{ Gender } \\
\hline Male & 852 & 54.3 \\
\hline Female & 716 & 45.7 \\
\hline \multicolumn{3}{|l|}{ Age } \\
\hline $18-40$ & 987 & 62.9 \\
\hline $41-60$ & 456 & 29.1 \\
\hline$>61$ & 125 & 8 \\
\hline \multicolumn{3}{|l|}{ Marital status } \\
\hline Married & 924 & 58.9 \\
\hline Widowed/divorced & 127 & 8.1 \\
\hline Single & 517 & 33 \\
\hline \multicolumn{3}{|l|}{ Income } \\
\hline First quintile & 335 & 21.4 \\
\hline Second quintile & 308 & 19.6 \\
\hline Third quintile & 429 & 12.7 \\
\hline Fourth quintile & 199 & 27.4 \\
\hline Fifth quintile & 297 & 18.9 \\
\hline \multicolumn{3}{|l|}{ Occupation } \\
\hline Employed & 571 & 36.4 \\
\hline Homemaker & 393 & 25.1 \\
\hline Student & 362 & 23.1 \\
\hline Unemployed & 242 & 15.4 \\
\hline \multicolumn{3}{|l|}{ Education (years) } \\
\hline $0-5$ & 197 & 12.6 \\
\hline $6-12$ & 670 & 42.7 \\
\hline$>12$ & 701 & 44.7 \\
\hline \multicolumn{3}{|l|}{ Physical activity } \\
\hline High & 535 & 34.1 \\
\hline Low & 1033 & 65.9 \\
\hline \multicolumn{3}{|l|}{ Smoking } \\
\hline Yes & 223 & 14.2 \\
\hline No & 1345 & 85.8 \\
\hline
\end{tabular}


$54.3 \%$ of the participants were male with the mean age of $36.92(S D=15.63), 45.7 \%$ were female with the mean age of $41.9(S D=15.15)$.

Table 2 shows the HRQoL scores as measured by SF36. The means (SD) of PCS and MCS were 73.39 (18.27) and 71 (15.92), respectively. $T$ test confirmed that the physical status of the participants was significantly more favorvable than their mental status $(P<0.01)$.

Table 3 compares the HRQoL dimensions between different demographic and socioeconomic groups. Females and elderly showed significantly lower scores in HRQoL and all subscales compared with males and young groups,
Table 2. The Participants' HRQoL Score Means

\begin{tabular}{lcc}
\hline Scales & Mean & SD \\
\hline PF & 85.63 & 22.3 \\
RP & 72.95 & 36.88 \\
BP & 73.14 & 26.42 \\
GH & 66.57 & 18.54 \\
VIT & 68.58 & 17.2 \\
SF & 78.88 & 19.785 \\
RE & 72.55 & 37.8 \\
MH & 68.36 & 16.7 \\
PCS & 73.39 & 18.27 \\
MCS & 71 & 15.92 \\
\hline
\end{tabular}

Abbreviation: HRQoL, health-related quality of life.

Table 3. The Mean (SD) of HRQoL Subscales Among Different Socio-Demographic Groups

\begin{tabular}{|c|c|c|c|c|c|c|c|c|c|}
\hline Variables (n & = 1568) & PF & RP & BP & GH & VIT & SF & RE & MH \\
\hline \multirow{2}{*}{ Gender } & Male & $\begin{array}{c}88.91 \\
(19.98)\end{array}$ & $\begin{array}{c}77.25 \\
(34.38)\end{array}$ & $\begin{array}{c}76.45 \\
(25.71)\end{array}$ & $\begin{array}{c}68.97 \\
(18.46)\end{array}$ & $\begin{array}{c}70.91 \\
(16.98)\end{array}$ & $\begin{array}{c}80.37 \\
(19.35)\end{array}$ & $\begin{array}{c}76.6 \\
(35.77)\end{array}$ & $\begin{array}{c}69.6 \\
(17.08)\end{array}$ \\
\hline & Female & $\begin{array}{c}81.74 \\
(24.23)\end{array}$ & $\begin{array}{c}67.84 \\
(39.07)\end{array}$ & $\begin{array}{c}69.19 \\
(26.72)\end{array}$ & $\begin{array}{c}63.7 \\
(18.25)\end{array}$ & $\begin{array}{c}65.81 \\
(17.07)\end{array}$ & $\begin{array}{c}77.11 \\
(20.15)\end{array}$ & $\begin{array}{c}67.72 \\
(39.65)\end{array}$ & $\begin{array}{c}66.88 \\
(16.12)\end{array}$ \\
\hline \multirow{3}{*}{ Age } & $18-40$ & $\begin{array}{c}92.05 \\
(16.64)\end{array}$ & $\begin{array}{l}81.814 \\
(30.60)\end{array}$ & $\begin{array}{c}77.99 \\
(25.15)\end{array}$ & $\begin{array}{c}70.28 \\
(17.56)\end{array}$ & $\begin{array}{c}71.21 \\
(16.61)\end{array}$ & $\begin{array}{c}80.85 \\
(19.09)\end{array}$ & $\begin{array}{c}77.64 \\
(34.23)\end{array}$ & $\begin{array}{c}70.17 \\
(16.42)\end{array}$ \\
\hline & $41-60$ & $\begin{array}{c}78.91 \\
(23.53)\end{array}$ & $\begin{array}{c}61.67 \\
(40.86)\end{array}$ & $\begin{array}{c}67.08 \\
(26.67)\end{array}$ & $\begin{array}{l}61.28 \\
(18.9)\end{array}$ & $\begin{array}{c}65.42 \\
(17.25)\end{array}$ & $\begin{array}{c}77.08 \\
(19.97)\end{array}$ & $\begin{array}{c}65.4 \\
(41.28)\end{array}$ & $\begin{array}{c}65.94 \\
(16.81)\end{array}$ \\
\hline & $>61$ & $\begin{array}{c}59.56 \\
(29.58)\end{array}$ & $\begin{array}{c}44.2 \\
(41.16)\end{array}$ & $\begin{array}{c}56.89 \\
(24.36)\end{array}$ & $\begin{array}{c}56.48 \\
(16.29)\end{array}$ & $\begin{array}{c}59.32 \\
(16.59)\end{array}$ & $\begin{array}{l}69.900 \\
(21.42)\end{array}$ & $\begin{array}{c}58.4 \\
(43.92)\end{array}$ & $\begin{array}{c}62.84 \\
(16.27)\end{array}$ \\
\hline \multirow{3}{*}{$\begin{array}{l}\text { Marital } \\
\text { status }\end{array}$} & Married & $\begin{array}{c}82.81 \\
(23)\end{array}$ & $\begin{array}{c}66.77 \\
(38.75)\end{array}$ & $\begin{array}{c}70.08 \\
(26.94)\end{array}$ & $\begin{array}{l}64.69 \\
(19.4)\end{array}$ & $\begin{array}{c}67.65 \\
(17.19)\end{array}$ & $\begin{array}{c}78.54 \\
(20.09)\end{array}$ & $\begin{array}{c}71.74 \\
(38.65)\end{array}$ & $\begin{array}{c}67.87 \\
(17.24)\end{array}$ \\
\hline & $\begin{array}{l}\text { Widowed or } \\
\text { divorce }\end{array}$ & $\begin{array}{l}75.59 \\
(30.6)\end{array}$ & $\begin{array}{c}67.91 \\
(38.81)\end{array}$ & $\begin{array}{c}69.85 \\
(26.62)\end{array}$ & $\begin{array}{c}61.06 \\
(16.54)\end{array}$ & $\begin{array}{c}66.25 \\
(18.27)\end{array}$ & $\begin{array}{c}75.19 \\
(21.47)\end{array}$ & $\begin{array}{c}67.97 \\
(40.36)\end{array}$ & $\begin{array}{l}65.51 \\
(16.2)\end{array}$ \\
\hline & Single & $\begin{array}{c}93.16 \\
(15.48)\end{array}$ & $\begin{array}{c}85.25 \\
(29.18)\end{array}$ & $\begin{array}{c}79.41 \\
(24.29)\end{array}$ & $\begin{array}{c}71.27 \\
(16.38)\end{array}$ & $\begin{array}{c}70.81 \\
(16.75)\end{array}$ & $\begin{array}{c}80.39 \\
(18.65)\end{array}$ & $\begin{array}{c}75.11 \\
(35.57)\end{array}$ & $\begin{array}{c}69.94 \\
(15.71)\end{array}$ \\
\hline \multirow{5}{*}{ Income } & First quintile & $\begin{array}{c}86.48 \\
(22.98)\end{array}$ & $\begin{array}{c}76.79 \\
(35.25)\end{array}$ & $\begin{array}{c}71.95 \\
(26.63)\end{array}$ & $\begin{array}{c}63.39 \\
(16.63)\end{array}$ & $\begin{array}{c}66.64 \\
(16.52)\end{array}$ & $\begin{array}{c}74.92 \\
(19.27)\end{array}$ & $\begin{array}{c}72.71 \\
(38.67)\end{array}$ & $\begin{array}{c}65.08 \\
(16.78)\end{array}$ \\
\hline & Second quintile & $\begin{array}{c}86.27 \\
(21.99)\end{array}$ & $\begin{array}{c}71.42 \\
(38.91)\end{array}$ & $\begin{array}{c}74.02 \\
(25.71)\end{array}$ & $\begin{array}{c}65.69 \\
(18.04)\end{array}$ & $\begin{array}{c}68.57 \\
(17.18)\end{array}$ & $\begin{array}{c}77.47 \\
(20.46)\end{array}$ & $\begin{array}{c}70.77 \\
(39.23)\end{array}$ & $\begin{array}{c}67.72 \\
(15.82)\end{array}$ \\
\hline & Third quintile & $\begin{array}{c}85.06 \\
(22.25)\end{array}$ & $\begin{array}{c}70.62 \\
(38.59)\end{array}$ & $\begin{array}{c}72.58 \\
(26.79)\end{array}$ & $\begin{array}{c}64.67 \\
(19.61)\end{array}$ & $\begin{array}{c}68.24 \\
(17.13)\end{array}$ & $\begin{array}{c}79.02 \\
(19.87)\end{array}$ & $\begin{array}{c}70.7 \\
(38.99)\end{array}$ & $\begin{array}{c}68 \\
(17.58)\end{array}$ \\
\hline & Fourth quintile & $\begin{array}{c}84.95 \\
(22.17)\end{array}$ & $\begin{array}{l}73.24 \\
(34.4)\end{array}$ & $\begin{array}{c}71.55 \\
(28.57)\end{array}$ & $\begin{array}{c}70.79 \\
(18.89)\end{array}$ & $\begin{array}{c}69.72 \\
(18.11)\end{array}$ & $\begin{array}{c}80.71 \\
(21.01)\end{array}$ & $\begin{array}{c}77.5544 \\
(32.63)\end{array}$ & $\begin{array}{l}71.39 \\
(17.2)\end{array}$ \\
\hline & Fifth quintile & $\begin{array}{c}85.34 \\
(22.11)\end{array}$ & $\begin{array}{c}73.4 \\
(35.46)\end{array}$ & $\begin{array}{c}75.42 \\
(24.81)\end{array}$ & $\begin{array}{c}70.95 \\
(18.09)\end{array}$ & $\begin{array}{c}70.52 \\
(17.32)\end{array}$ & $\begin{array}{c}83.37 \\
(17.61)\end{array}$ & $\begin{array}{c}73.51 \\
(36.88)\end{array}$ & $\begin{array}{c}71.19 \\
(15.09)\end{array}$ \\
\hline \multirow{4}{*}{ Occupation } & Employed & $\begin{array}{c}90.32 \\
(17.33)\end{array}$ & $\begin{array}{c}78.06 \\
(32.83)\end{array}$ & $\begin{array}{c}78.5 \\
(24.61)\end{array}$ & $\begin{array}{c}70.56 \\
(18.99)\end{array}$ & $\begin{array}{c}72.27 \\
(17.11)\end{array}$ & $\begin{array}{c}82.37 \\
(19.05)\end{array}$ & $\begin{array}{c}77.4 \\
(34.55)\end{array}$ & $\begin{array}{c}71.1 \\
(17.32)\end{array}$ \\
\hline & Homemaker & $\begin{array}{c}76.03 \\
(26.28)\end{array}$ & $\begin{array}{c}59.98 \\
(41.07)\end{array}$ & $\begin{array}{c}62.74 \\
(27.25)\end{array}$ & $\begin{array}{c}59.15 \\
(17.41)\end{array}$ & $\begin{array}{l}63.24 \\
(16.4)\end{array}$ & $\begin{array}{c}75.12 \\
(19.94)\end{array}$ & $\begin{array}{c}63.76 \\
(42.21)\end{array}$ & $\begin{array}{c}64.49 \\
(15.49)\end{array}$ \\
\hline & Student & $\begin{array}{c}93.74 \\
(15.05)\end{array}$ & $\begin{array}{c}84.59 \\
(30.05)\end{array}$ & $\begin{array}{c}80.44 \\
(23.09)\end{array}$ & $\begin{array}{c}72.13 \\
(16.24)\end{array}$ & $\begin{array}{c}71.98 \\
(16.37)\end{array}$ & $\begin{array}{c}80.28 \\
(18.09)\end{array}$ & $\begin{array}{c}75.41 \\
(35)\end{array}$ & $\begin{array}{l}71.86 \\
(14.9)\end{array}$ \\
\hline & Unemployed & $\begin{array}{c}78.08 \\
(26.51)\end{array}$ & $\begin{array}{c}64.56 \\
(39.86)\end{array}$ & $\begin{array}{c}66.44 \\
(27.03)\end{array}$ & $\begin{array}{c}60.85 \\
(16.96)\end{array}$ & $\begin{array}{c}63.45 \\
(16.45)\end{array}$ & $\begin{array}{c}74.63 \\
(21.82)\end{array}$ & $\begin{array}{c}71.07 \\
(39.46)\end{array}$ & $\begin{array}{c}62.92 \\
(17.07)\end{array}$ \\
\hline \multirow{3}{*}{ Education (y) } & $0-5$ & $\begin{array}{c}66.68 \\
(29.54)\end{array}$ & $\begin{array}{c}51.9 \\
(42.4)\end{array}$ & $\begin{array}{c}57.12 \\
(26.92)\end{array}$ & $\begin{array}{c}53.83 \\
(16.92)\end{array}$ & $\begin{array}{c}57.81 \\
(16.45)\end{array}$ & $\begin{array}{c}70.05 \\
(21.85)\end{array}$ & $\begin{array}{c}57.19 \\
(44.03)\end{array}$ & $\begin{array}{c}59.59 \\
(16.19)\end{array}$ \\
\hline & $5-12$ & $\begin{array}{c}86 \\
(21.35)\end{array}$ & $\begin{array}{c}72.35 \\
(37.25)\end{array}$ & $\begin{array}{c}70.99 \\
(25.63)\end{array}$ & $\begin{array}{c}64.7 \\
(17.52)\end{array}$ & $\begin{array}{c}67.75 \\
(16.18)\end{array}$ & $\begin{array}{c}77.79 \\
(18.68)\end{array}$ & $\begin{array}{c}71.13 \\
(38.95)\end{array}$ & $\begin{array}{c}67.08 \\
(16.35)\end{array}$ \\
\hline & $5-12$ & $\begin{array}{c}90.62 \\
(17.53)\end{array}$ & $\begin{array}{c}79.45 \\
(32.38)\end{array}$ & $\begin{array}{c}79.73 \\
(24.73)\end{array}$ & $\begin{array}{c}71.92 \\
(17.84)\end{array}$ & $\begin{array}{c}72.4 \\
(16.99)\end{array}$ & $\begin{array}{c}82.4 \\
(19.32)\end{array}$ & $\begin{array}{c}78.22 \\
(33.35)\end{array}$ & $\begin{array}{c}72.04 \\
(16.08)\end{array}$ \\
\hline \multirow{2}{*}{ Smoking } & Yes & $\begin{array}{l}84.48 \\
(22.6)\end{array}$ & $\begin{array}{l}66.031 \\
(37.56)\end{array}$ & $\begin{array}{c}70.18 \\
(27.62)\end{array}$ & $\begin{array}{c}63.67 \\
(18.63)\end{array}$ & $\begin{array}{c}64.95 \\
(17.54)\end{array}$ & $\begin{array}{c}76.45 \\
(20.63)\end{array}$ & $\begin{array}{c}65.47 \\
(39.75)\end{array}$ & $\begin{array}{c}64.46 \\
(16.43)\end{array}$ \\
\hline & No & $\begin{array}{c}85.83 \\
(22.25)\end{array}$ & $\begin{array}{c}74.1 \\
(36.66)\end{array}$ & $\begin{array}{c}73.63 \\
(26.19)\end{array}$ & $\begin{array}{c}67.05 \\
(18.49)\end{array}$ & $\begin{array}{c}69.18 \\
(17.08)\end{array}$ & $\begin{array}{c}79.28 \\
(19.61)\end{array}$ & $\begin{array}{l}73.72 \\
(37.4)\end{array}$ & $\begin{array}{c}69 \\
(16.66)\end{array}$ \\
\hline \multirow{2}{*}{$\begin{array}{l}\text { Physical } \\
\text { activity }\end{array}$} & High & $\begin{array}{c}89.11 \\
(19.14)\end{array}$ & $\begin{array}{c}74.9 \\
(35.15)\end{array}$ & $\begin{array}{c}77.92 \\
(25.25)\end{array}$ & $\begin{array}{c}70.09 \\
(18.33)\end{array}$ & $\begin{array}{c}70.97 \\
(16.47)\end{array}$ & $\begin{array}{c}81.93 \\
(19.39)\end{array}$ & $\begin{array}{c}74.76 \\
(35.98)\end{array}$ & $\begin{array}{c}69.6 \\
(16.28)\end{array}$ \\
\hline & Low & $\begin{array}{c}83.84 \\
(23.58)\end{array}$ & $\begin{array}{c}71.95 \\
(37.73)\end{array}$ & $\begin{array}{c}70.66 \\
(26.68)\end{array}$ & $\begin{array}{l}64.74 \\
(18.4)\end{array}$ & $\begin{array}{c}67.34 \\
(17.45)\end{array}$ & $\begin{array}{l}77.29 \\
(19.8)\end{array}$ & $\begin{array}{c}71.4 \\
(38.73)\end{array}$ & $\begin{array}{c}67.71 \\
(16.89)\end{array}$ \\
\hline
\end{tabular}


respectively. In addition, married individuals expressed lower scores in all HRQoL subscales but RE compared with single subjects. Unemployed subjects showed significantly lower HRQoL compared with employed group as well as students. Significantly higher HRQoL score was observed among fifth quintile income group and individuals with $>12$ years of education $(P<0.01)$. In addition, smoking individuals expressed significantly lower HRQoL as compared with non-smoking subjects $(P<0.05)$. Ultimately, HRQoL was significantly higher among physically active groups compared with inactive group $(P<0.05)$.

Table 4 presents the results of multiple logistic regression analysis. Accordingly, age, gender, education level, physical activity, and smoking habit are identified as the significant determinants of both PCS and MCS. In addition, while income does shows no significant impact on PCS, low income is associated with MCS.

\section{Discussion}

Our results identified a moderate level of HRQoL among the study sample. The SF-36-based HRQoL scores in this study agree with those reported from other studies conducted in Tehran. ${ }^{5,7}$ Females, elderly, widowed or divorced individuals, low income groups, and unemployed, low education level, low physical activity, and smoking individuals were accompanied by lower scores in most of HRQoL subscales. Consistently, previous studies in Iran and other countries also report relatively low HRQoL among females, ,7-12 high age subjects, ,4,5,7, 10,12-14 widowed or divorced subjects, ${ }^{4,12,13}$ and low income, ${ }^{9,12}$ unemployed, ${ }^{4,12}$ physical inactivity, ${ }^{15,16}$ and smoking ${ }^{8,17}$ groups.

We also used logistic regression to gain a clearer insight into the determinants of HRQoL. The results of logistic regression showed that gender, age, education, occupation, smoking, and physical activity predicts both PCS and MCS.

Aghamolaei et al, ${ }^{5}$ identified age as a direct predictor of HRQoL, especially in terms of PCS. The pattern of age distribution among Iranian population is rapidly changing; it has been forecasted that the elderly population will account for $10.5 \%$ of population by 2025 and $21.1 \%$ by $2050 .{ }^{18}$ Considering this growing trend, our results point towards the urgent need for focused attention to health issues in this age group.

We also found significantly lower HRQoL among females in terms of both PCS and MCS. Thus, women are more vulnerable to the risk factors of low HRQoL. At the same time, less educated subjects displayed poorer HRQoL in both PCS and MCS terms. Also our survey showed relatively low PCS and MCS scores for unemployed individuals and homemakers. Similar results was observed from a previous study in Iran. ${ }^{5}$ These findings are not surprising since the vulnerability of individuals to mental and physical problems increases when unemployed and most home-

Table 4. The Results of Logistic Regression Analysis

\begin{tabular}{|c|c|c|c|c|c|}
\hline \multirow[t]{2}{*}{ Variable } & & \multicolumn{2}{|c|}{ PCS } & \multicolumn{2}{|c|}{ MCS } \\
\hline & & OR & $95 \% \mathrm{Cl}$ & OR & $95 \% \mathrm{Cl}$ \\
\hline \multirow{2}{*}{ Gender } & Male & 1 & & 1 & \\
\hline & Female & $1.51^{\mathrm{a}}$ & $1.23-1.83$ & $1.35^{\mathrm{a}}$ & $1.1-1.66$ \\
\hline \multirow{3}{*}{ Age } & $18-40$ & 1 & & 1 & \\
\hline & $41-60$ & $2.38^{a}$ & $1.82-3.1$ & $1.56^{\mathrm{a}}$ & $1.21-2.01$ \\
\hline & $>61$ & $5.48^{a}$ & $4.17-7.21$ & $2.17^{\mathrm{a}}$ & $1.62-2.91$ \\
\hline \multirow{3}{*}{ Marriage } & Single & 1 & & 1 & \\
\hline & Married & 1.34 & $1.04-1.74$ & 0.91 & $0.691-1.182$ \\
\hline & Widowed or divorced & 1.24 & $1.02-1.51$ & 0.87 & $0.559-1.347$ \\
\hline \multirow{3}{*}{$\begin{array}{l}\text { Education } \\
\text { (year) }\end{array}$} & $>12$ & 1 & & 1 & \\
\hline & $6-12$ & $1.26^{a}$ & $1.06-1.51$ & $1.3^{a}$ & $1.15-1.4$ \\
\hline & $0-5$ & $2.23^{a}$ & $1.69-2.95$ & $2.18^{a}$ & $1.87-2.53$ \\
\hline \multirow{4}{*}{ Occupation } & Employed & 1 & & 1 & \\
\hline & Homemaker & $1.76^{a}$ & $1.3-2.39$ & $1.38^{a}$ & $1.11-1.71$ \\
\hline & Student & 1.39 & $0.98-1.96$ & 1.16 & $0.84-1.6$ \\
\hline & Unemployed & $2.13^{a}$ & $1.64-2.78$ & $1.49^{a}$ & $1.16-1.92$ \\
\hline \multirow{5}{*}{ Income } & First quintile & 0.64 & 0.403-1.03 & $1.32^{\mathrm{a}}$ & $1.09-1.61$ \\
\hline & Second quintile & 0.83 & $0.551-1.26$ & 1.27 & $0.92-1.74$ \\
\hline & Third quintile & 0.98 & $0.67-1.414$ & $1.46^{b}$ & $1.02-2.09$ \\
\hline & Fourth quintile & 1.16 & 0.84-1.59 & 1.12 & $0.8-1.56$ \\
\hline & Fifth quintile & 1 & & 1 & \\
\hline \multirow{2}{*}{ Physical activity } & Yes & 1 & & 1 & \\
\hline & No & $1.39^{a}$ & $1.09-1.77$ & $1.39^{a}$ & $1.11-1.73$ \\
\hline \multirow{2}{*}{ Smoking habit } & Yes & $1.58^{a}$ & $1.24-2.03$ & $1.47^{a}$ & $1.15-1.89$ \\
\hline & No & 1 & & 1 & \\
\hline
\end{tabular}

a $P<0.01 ;{ }^{b} P<0.05$ 
makers are female which were already shown to have lower HRQoL.

On the other hands, we identified no significant relationship between income level, and PCS; however, low income showed signals of association with lower MCS. Therefore, this may be concluded that high income would not necessarily correlate with high HRQoL, however, low income may negatively influence the mental aspect of QoL. This observation can be explained by the fact that adequate income is important not only to cover and meet the basic needs of life, but it is also a very important to maintain psychological health. ${ }^{18}$

Two life-style factors including smoking habit and physical inactivity we found to positively and negatively impact HRQoL, respectively. Similarly, using SF-12 questionnaire, Dey et al identified smoking as a negative predictor of HRQoL in young Swiss men. ${ }^{19}$ Others have also reported similar results. ${ }^{17,20}$ Furthermore, there is evidence to show that physical activity could result in better HRQoL, ${ }^{19,20}$ confirming our observations.

In general, our results replicated identification of risk factors of HRQoL reported in previous studies. In particular female and elderly are more prone to low HRQoL and are the prime groups to be focused in intervention programs. The observed impact of education level on HRQoL suggests that promotion of both public and academic education would enhance both public health and HRQoL. Public education focused at improving life style may also yield additional health-related outcomes by encouraging individuals to leave unhealthy habits such as smoking and go through healthy activities such as sport or meditation.

\section{Study Limitations}

Cross-sectional studies have limited ability to capture the cause and effect relationship between variable. Therefore further longitudinal studies on the HRQoL are recommended. Also, we did not adjust the existence of possible chronic conditions in our sample. Although our results were based on a relatively large sample and the study was conducted in one of the largest Iranian metropolises, the determinant factors of $\mathrm{HRQ}$ oL identified may not be representative of Iranian population, particularly the rural areas. Therefore, while our findings have the potential to give a glimpse of HRQoL in Iranian large cities, caution must me exercised in generalization of the results for small cities and villages. At the same time, our results encourage conduction of large-scale studies enabling incorporation of HRQoL concept into national policy-making.

\section{Conclusions}

This study explored the HRQoL among population of an
Iranian metropolis, Shiraz, The capital of Fars province. $\mathrm{HRQ}$ oL was found to be at moderate level. Females, elderly, low income groups, and unemployed, low education level, low physical activity, and smoking individuals showed relatively low scores in some or all of HRQoL dimensions. Our results may be useful in developing provincial-level programs to improve HRQoL and encourages further conduction of large-scale studies enabling incorporation of $\mathrm{HRQ}$ oL concept into national policy-making.

\section{Abbreviations}

(HRQoL): Health-related quality of life.

\section{Authors' Contributions}

SV and MA jointly devised the study and made the major contribution in analysis of the data. AR, ZK, and SL took part in data analysis and interpretation of the results. MA drafted and revised the manuscript. All authors read and approved the final manuscript.

\section{Competing Interests}

The authors declared no financial disclosure.

\section{Acknowledgements}

This study was a research project supported by Shiraz University of Medical Science. The authors would like to thank Ms. A. Keivanshekouh from the Research Improvement Center of Shiraz University of Medical Sciences helping us in English editing of the manuscript.

\section{References}

1. World Health Organization. The world health report: health systems financing: the path to universal coverage. Geneva: WHO; 2010.

2. Stiefel MC, Perla RJ, Zell BL. A healthy bottom line: healthy life expectancy as an outcome measure for health improvement efforts. Milbank Q. 2010;88(1):30-53. doi:10.1111/j.1468-0009.2010.00588.x.

3. Labbe JA. Health-Adjusted Life Expectancy: Concepts and Estimates. In: Preedy VR, Watson RR, eds. Handbook of Disease Burdens and Quality of Life Measures. New York: Springer; 2010

4. Sabbah I, Drouby N, Sabbah S, Retel-Rude N, Mercier M. Quality of Life in rural and urban populations in Lebanon using SF-36 Health Survey. Health Qual Life Outcomes. 2003;1:30. doi:10.1186/1477-7525-1-30.

5. Aghamolaei T, Sadat Tavafian S, Zare S. Determinants of health related quality of life on people living in Bandar Abbas, Iran. Iran J Publ Health. 2011;40(2):128-135.

6. Wilson I, Cleary P. Linking clinical variables with health related quality of life. A conceptual model of patient 
outcomes. JAMA. 1995;273(1):59-65.

7. Montazeri A, Goshtasebi A, Vahdaninia M, Gandek B The Short Form Health Survey (SF-36): translation and validation study of the Iranian version. Qual Life Res. 2005;14(3):875-882.

8. Mohammadpour R. Association between lifestyle satisfaction and tendency to behavioral change with health related quality of life among 40 years old and over in (North of Iran) Mazandaran. Iran J Health Sci. 2013;1(1):31-35.

9. Khader S, Hourani M, Al-Akour N. Normative data and psychometric properties of short form 36 health survey (SF-36, version 1.0) in the population of north Jordan. East Mediterr Health. 2011;17(5):368-74.

10. Li L, Wang H, Shen Y. Chinese SF-36 Health Survey: translation, cultural adaptation, validation, and normalisation. $\mathrm{J}$ Epidemiol Community Health. 2003;57(4):259-263.

11. Lahelma E, Martikainen P, Rahkonen O, Roos E Saastamoinen P. Occupational class inequalities across key domains of health: results from the Helsinki Health Study. Eur J Public Health. 2005;15(5):504-510.

12. Butterworth $\mathrm{P}$, Crosier $\mathrm{T}$. The validity of the SF-36 in an Australian National Household Survey: demonstrating the applicability of the Household Income and Labour Dynamics in Australia (HILDA) Survey to examination of health inequalities. BMC Public Health. 2004;4:44 doi:10.1186/1471-2458-4-44

13. Ghafari R, Rafiei M, Taheri-Nejad M. Assessment of health related quality of life by SF-36 version 2 in general population of Qom city (Persian). Arak Medical University Journal. 2014;16(11):63-72.
14. Nejati V, Ahmadi K, Sharifian M, Shoaee F. Comparing quality of life dimension in different age decades: prediction for aging (Persian). Journal of Fasa University of Medical Sciences. 2010;11(4):227-32.

15. Farid M, Dabiran S. Health-related quality of life in iranian women with different levels of physical activity. Asian $\mathrm{J}$ Sports Med. 2012;3(3):203-207.

16. Laforge RG, Rossi JS, Prochaska JO, Velicer WF, Levesque DA, McHorney CA. Stage of regular exercise and health-related quality of life. Prev Med. 1999;28(4):349-360

17. Tillmann M, Silcock J. A comparison of smokers' and ex-smokers' health-related quality of life. J Public Health Med. 1997;19(3):268-273.

18. Tajvar M, Arab M, Montazeri A. Determinants of healthrelated quality of life in elderly in Tehran, Iran. BMC Public Health. 2008;8:323. doi:10.1186/1471-2458-8-323.

19. Dey M, Gmel G, Mohler-Kuo M. Body mass index and health-related quality of life among young Swiss men. BMC Public Health. 2013;13:1028. doi:10.1186/14712458-13-1028.

20. Kearns B, Ara R, Young T, Relton C. Association between body mass index and health-related quality of life, and the impact of self-reported long-term conditions - crosssectional study from the south Yorkshire cohort dataset. BMC Public Health. 2013;13:1009. doi:10.1186/1471-

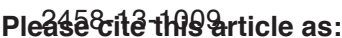

Vahedi S, Rezapour A, Arefnezhad M, Lak S, Bastami F. Identification of determinant factors of health-related quality of life in iranian urban population. Int J Hosp Res. 2016;5(1):3540. doi:10.15171/ijhr.2016.07. 